\title{
Finanzkrise als Schocktherapie
}

Noch ist die Reichweite der Finanzmarktkrise schwer zu bestimmen. ${ }^{1}$ Die Erschütterungen, die von ihr in den Banketagen und zunehmend auch in der Realwirtschaft hervorgerufen werden, lassen viele an die Weltwirtschaftskrise der Jahre nach 1929 denken. Aber auch denjenigen, die derartige historische Einordnungen nicht gelten lassen wollen, drängt sich die Einschätzung auf, dass "die Welt nach dieser Krise eine andere" sein wird (Peer Steinbrück). Der neoliberale Fundamentalismus, demzufolge die Marktkräfte in anonymer Selbstregulation die bestmögliche wirtschaftliche und politische Steuerung sichern und zur Wohlfahrt aller führen, hatte durch die unübersehbare Zunahme von Einkommensungleichheit und Armut schon zuvor an Überzeugungskraft eingebüßt. Nun könnte er seine Glaubwürdigkeit nachhaltig verspielt haben. Denn das zumindest hat der Schock der Finanzkrise bewirkt: Die Botschaft der „you're on your own“Welt mit ihrer hochmütigen Verachtung staatlicher Aktivitäten und Institutionen klingt jetzt wie Satire, nachdem eben noch Undenkbares Realität geworden ist. Ein Weltbild stürzte ein. Wie wird diese Situation verarbeitet? Welche Neujustierung erfährt das Verhältnis von Markt und Staat?

Viele haben sich in den vergangenen Jahren die Finger wund geschrieben gegen die Glücksversprechungen der Marktradikalen. Es mangelte nicht an Gefahrenprognosen. Der zum Erfolgsmodell stilisierte und als Maßstab gehandelte anglo-amerikanische Weg wurde in vielen seiner Negativwirkungen ausgeleuchtet: bezüglich der Gefahren, die von einer finanzmarktorientierten Wirtschaft national und international ausgehen; der Herausbildung einer immer mehr in Arm und Reich zerfallenden Gesellschaft; dem verstärkten Auftreten von staatlich nicht oder nur ganz unzureichend verarbeiteten Umweltproblemen; der Tendenz zu einer verrottenden Infrastruktur und fehlgeleiteter Energiegewinnung usw.
Heute können wir nur konstatieren: Was noch so überzeugende Argumente nicht geschafft haben, ist durch die Finanzkrise mit einem Schlag eingetreten. Fast über Nacht gerät eine Ideologie ins Wanken, die in den letzten Dekaden durch die Hegemonie des „Finanz-Ökonomie-Komplexes" - unterstützt durch ein publizistisches Trommelfeuer ohnegleichen - ihre Meinungsführerschaft zementieren konnte. Ihre simple Botschaft, „mehr Markt, weniger Staat" wurde damit gegen alle noch so gut begründeten Einwände immunisiert und politisch in Szene gesetzt. Deregulierung des Kapitalverkehrs, Privatisierung der öffentlichen Dienstleistungen, Alleinzuständigkeit bei Arbeitssuche und Umstellung der Altersvorsorge auf kapitalbasierte Systeme - immer galt als Prämisse: Der Staat, die öffentlich getragene Intervention, ist dem Markt unterlegen und stört nur die effizienten Lösungen.

Heute wissen (fast) alle, dass diese Rechnung nicht aufgeht. Auch der trotzige Aufmacher „Wir letzten Neoliberalen“, unter dem im Handelsblatt vom 22.10.2008 die Rückbesinnung auf die Prinzipien der Marktwirtschaft beschworen wird, und die nach kurzer Sendepause neu ertönenden Appelle, „die Finanz- und Wirtschaftskrise darf nicht zur Systemfrage werden“ (Reiner Brüderle, FAS, 02.11.2008), werden die Delegitimierung alleiniger Marktsteuerung und die Entmystifizierung seiner Heilskräfte kaum mehr rückgängig machen können. Damit liegt in der Tat die Frage auf dem Tisch, „was macht eine Gesellschaft, die gerade die Zerstörung ihrer Lebenssicherheit erlebt?" (Frank Schirrmacher, FAZ, 01.11.2008), weil ihre bestimmenden ökonomisch-politischen Gestaltungsprinzipien versagt haben.

Der Bankrott eines imperialen Gestaltungsanspruchs rückt die Frage ins Zentrum, wie handhabbare alternative Konzepte entwickelt und umgesetzt werden könnten. Mit größter Dinglichkeit muss es der Politik mit dem Zusammenbruch der
Finanzmärkte und dem Nachweis, dass die Selbstheilungskräfte des Marktes nichts hergeben zur Überwindung der Krise, darum gehen, angemessene, erfolgversprechende Maßnahmen zu entwickeln. Die anstehenden Aufgaben werfen neue Unsicherheiten auf. Vier damit verbundene Fragen seien zur Sprache gebracht:

(1) Weitgehende Einigkeit besteht darin, dass ohne eine globale Finanzarchitektur, mit der in supranationalen Strukturen eine Regulierungsform oberhalb der nationalen Interessen erwirkt werden kann, keine tragfähigen Lösungen mehr möglich sind. Dieser Sachverhalt erschwert eine Orientierung an den vorliegenden historischen Erfahrungen. Denn die Lehre aus der Weltwirtschaftskrise 1929, dass das Verhältnis von Staat und Markt neu gefasst werden muss und neue Stabilität und insbesondere ein hoher Beschäftigungsstand nur durch gezielte Fiskal- und Geldpolitik erreicht werden könne, begründet zunächst nationale Interventionsprogramme. Die Frage

\footnotetext{
Einschätzungen zu den Folgen der Finanzmarktkrise stehen zurzeit unter dem Vorbehalt einer sich ständig verändernden Real- und Informationslage. Redaktionsschluss für diesen Text war der 3.11.08.
}

Peter Kalmbach, Prof. Dr. oec. publ., war bis 2005 Professor an der Wirtschaftswissenschaftlichen Fakultät der Universität Bremen. Arbeitsschwerpunkte: Einkommensverteilung, technischer und struktureller Wandel, Arbeitsmarkt und wirtschaftliches Wachstum. e-mail:pkalm@uni-bremen.de Michael Schumann, Prof. Dr., Präsident des Soziologischen Forschungsinstituts e. V. an der Universität Göttingen. Arbeitsschwerpunkte: Industrie- und Arbeitssoziologie, Industrielle Beziehungen. e-mail: michael.schumann@sofi. uni-goettingen.de 
heißt heute: Wie sind die Keynesschen Konzepte einer Vollbeschäftigungspolitik in eine globalisierte Ökonomie zu übersetzen? Für welche Lebensrisiken seiner Bürger soll und kann der Staat unter diesen Bedingungen Zuständigkeit übernehmen und in welcher Form? Wie sieht ein entsprechendes internationales Politikprogramm aus, das nur funktionieren kann, wenn es die unterschiedlichen nationalen Interessen konsensual auszutarieren vermag?

(2) Der anstehende wirtschaftlich-politische Konzeptwechsel fordert innovative Lösungen. Es ist also die Fähigkeit und Bereitschaft zu neuem Denken gefragt. Kann diese Aufgabe gelingen mit denjenigen Akteuren als einschlägige Experten, die die Misere zum Teil mitverschuldet haben? Werden da nicht Brandstifter zu Feuerwehrleuten, denen weder Offenlegung der Schuldzusammenhänge und Strukturdefizite noch radikale Erneuerung naheliegen? Umgekehrt mag auch die Skepsis begründet sein, dass einem gegenüber Wirtschaftsinteressen eher subaltern und devot auftretenden politischen Personal keine überzeugenden Steuerungsleistungen zuzutrauen sind, weil diese Vertreter, wie es der US-Wirtschaftsexperte David M. Smick ausdrückt, „nicht klug genug sind, um sich gegen die smarten Wall-Street-Boys mit ihren cleveren Anwälten zu behaupten “ (FR 31.10.2008). Kann der Rat und das Engagement jener mobilisiert werden, die vorab vor den Systemdefekten des Finanzmarktkapitalismus gewarnt haben? Gelingt es, kompetente, innovative und dabei nicht vorbelastete Gestalter und Umsetzer zu rekrutieren?

(3) Inhaltlich geht es im Kern der Lösungskonzepte um mehr Staat und Regulierung. Die Gefahren, dass dabei administrativ entwickelte und praktizierte Politikansätze herauskommen, werden damit deutlich erhöht. Schon die Kontrolle durch die Parlamente (noch dazu bei Zeitdruck) kommt beim Aufspannen der großen Schutzschirme für die Finanzwelt unter die Räder von basisnahen demokratischen Rückbezügen ganz zu schweigen. Gerade wenn in der Bankenkrise auch deutlich wurde, dass die geltenden Handlungsanreize der Finanzmärkte dazu führten, dass die lokalen und regionalen Aufgaben vernachlässigt wurden und entsprechende Defizite entstanden, so muss für alle neuen Regulierungs- und Organisationskonzepte eine erweiterte Sensibilität geltend gemacht werden für transparente, kontrollierbare Lösungen. Bei allen Zwängen zu zentralistischen Strukturen ist immer ein Mehr an Rückkopplung an die betroffenen Akteursebenen mitzudenken. Damit ist als Anforderung gestellt: Wie sind bei Zentralisierungskonzepten auch Dezentralisierungsansprüche einzulösen?

Die Schwierigkeiten bei der Lösungssuche erscheinen noch größer, wenn die historischen Erfahrungen mit dem Staat als Gestaltungsakteur einbezogen werden. Keineswegs sichern, wie wir wissen, die vom Staat generierten Maßnahmen das Primat gesellschaftlicher Rationalität. Insofern muss für die anstehenden Aufgaben auch der Staat ein Stück weit neu erfunden werden. Vor allem ist zu fragen: Wie lassen sich Akteure mobilisieren, seien es Parteien, Gewerkschaften oder Bürgerbewegungen, die ein Staatshandeln absichern oder gar erzwingen, das dem erforderlichen neuen Rollenprofil entspricht?

(4) Erkennbar schlägt die Finanzkrise auf die Realökonomie durch. Um der erschwerten Wettbewerbssituation gerecht werden zu können, wird in der Wirtschaft bereits ein neuer Schub betrieblicher Rationalisierung angekündigt. Dabei besteht die Gefahr, dass wiederum auf jene Instrumentarien zurückgegriffen wird, die unter der Benchmark-Dominanz des anglo-amerikanischen Modells als erfolgversprechende Unternehmensstrategien gehandelt wurden. In der vom Finanzmarkt dominierten Denkweise und dem daraus abgeleiteten
Shareholder-Value-Konzept und seiner Kurzfristökonomie ist eine systematische Vernachlässigung der Human-Ressourcen erfolgt. Weil sich der Shareholder-Ansatz mehr auf die Einlösung von Finanzanforderungen als auf erfolgversprechende Produktionskonzepte konzentrierte, geriet der notwendige Aufbau, die Pflege und Qualifizierung der Arbeitskräfte allzu oft ins Abseits. Die Konkurrenzfähigkeit der exportorientierten deutschen Industrie hängt jedoch insbesondere von ihrer Reaktionsfähigkeit auf Veränderungen und von ihrer Innovationskraft ab. Diese Herausforderungen sind nur zu bestehen, wenn das Know-how der Beschäftigten bestmöglich entwickelt und umfassend genutzt wird. Das System der deutschen industriellen Beziehungen ermöglicht es, die Kompetenzen der Beschäftigten in die Entwicklung der Unternehmensstrategien produktiv einzubringen und konsensuale Umsetzungsformen zu suchen. Wird es also gelingen, einen arbeitspolitischen Paradigmenwechsel durchzusetzen, der die unter den traditionellen, tayloristisch-toyotistischen Vorzeichen vergeudeten Handlungs- und Verhaltenspotenziale tatsächlich erschließt und im gemeinsamen Interesse von Unternehmen und Beschäftigten fruchtbar macht?

Die Weltwirtschaftskrise von 1929 hat schließlich den „New Deal“ hervorgebracht. Was sich aus dem Crash der Finanzmärkte 2008 entwickeln wird, kann man gegenwärtig kaum prognostizieren. Wünschen würden wir uns, dass er zumindest „New Reflection“ auslöst. Gerade für Europa könnte eine große Chance darin liegen, mit der Überwindung der Finanzkrise Schubkraft zu gewinnen für Lösungsstrategien, die das Gesamtpaket der zu gestaltenden Aufgaben in neuer Qualität angehen. Nicht also als Deregulierungsgemeinschaft, sondern in verbesserter transnationaler Durchsetzung einer gesellschaftlicher Vernunft verpflichteten Politik. 